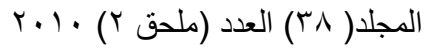
(ISSN 1815-316x)
مجلة زر اعة الر افدين

استخدام الفحم النباتي في تقليل الإصابة بالفطر Fusarium oxysporum f.sp.capsici المسبب لمرض ذبول القلفل

\author{
علاء حميذ محمد \\ نضال بونس محمد \\ قسم وقاية النبات / كلية الزر اعة و الغابات علدات
}

الخلاصة

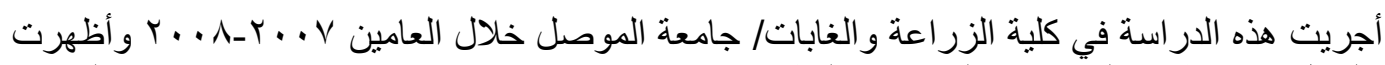

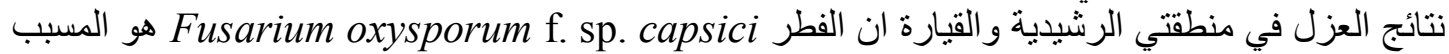

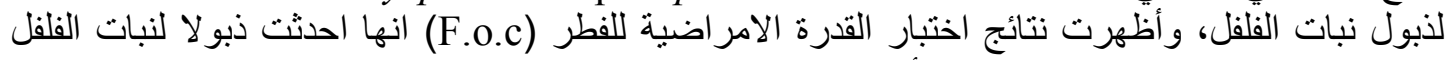

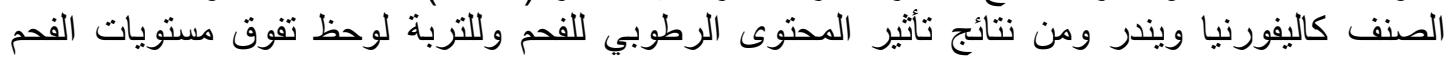

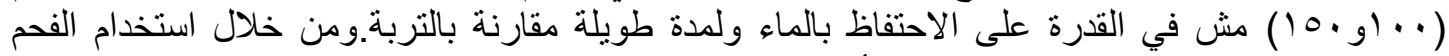

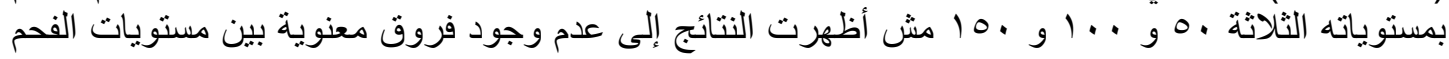

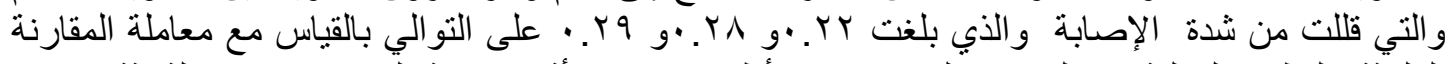

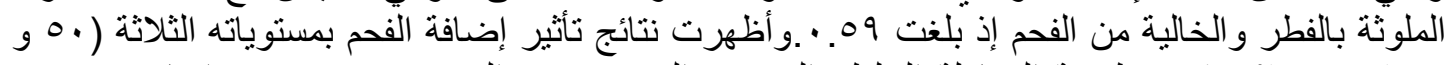

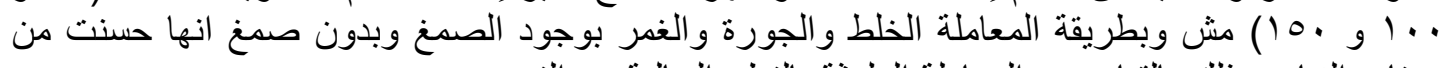

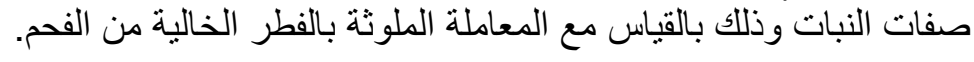

المقدمة

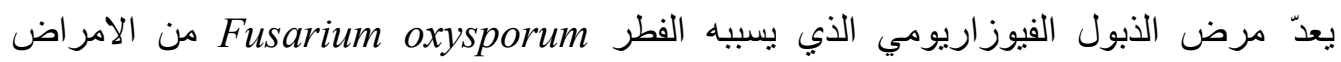

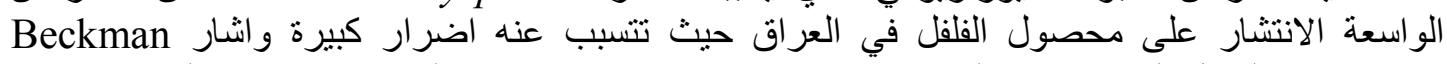

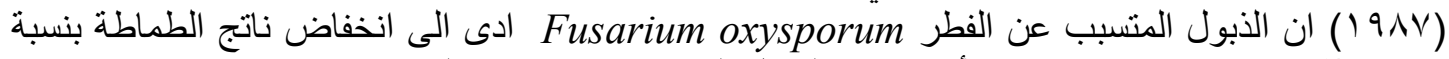

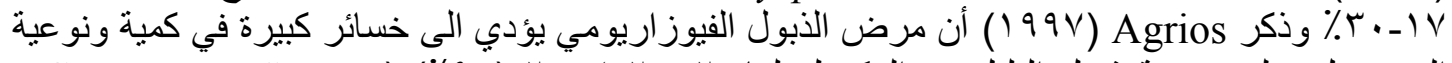

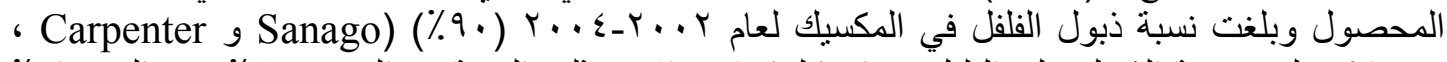

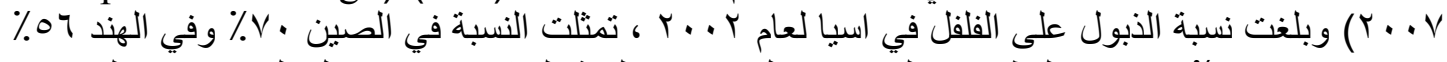

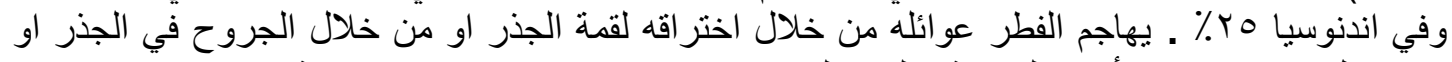

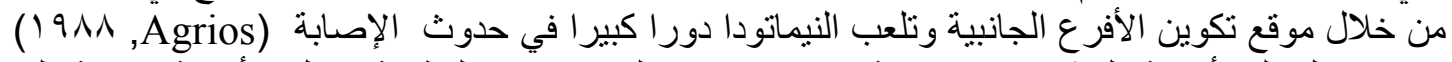

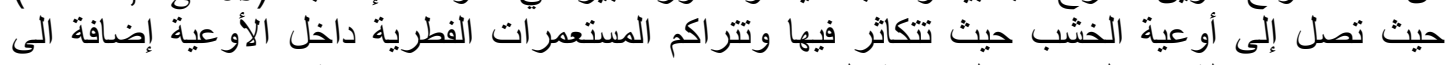

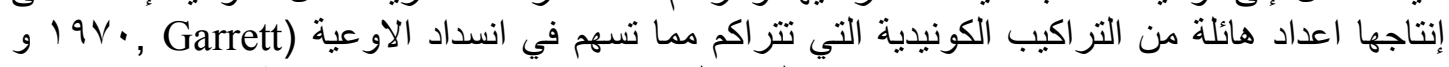

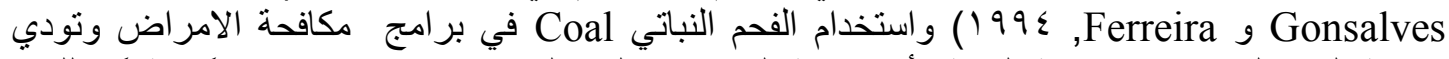

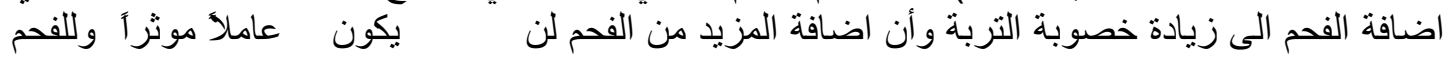

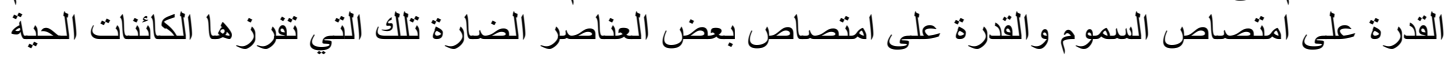

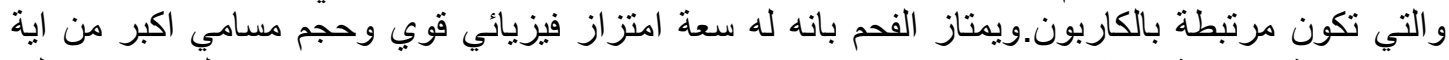

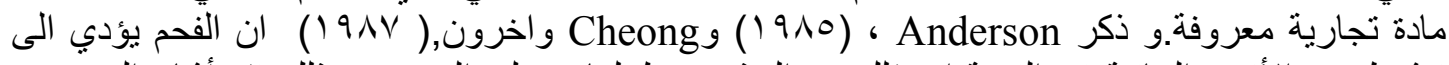

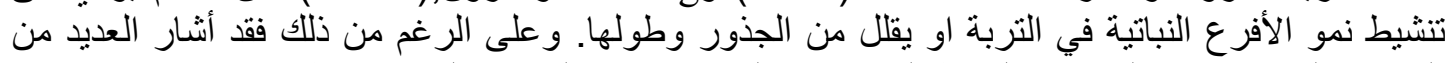

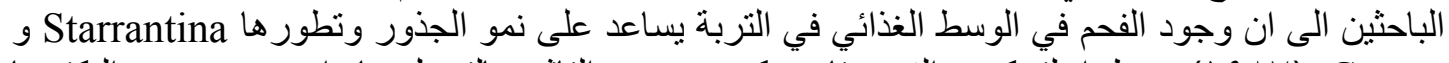
والفطرئ Carusa

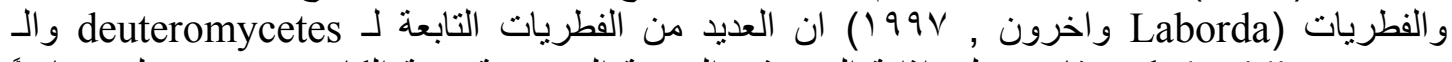
basidiomycetes

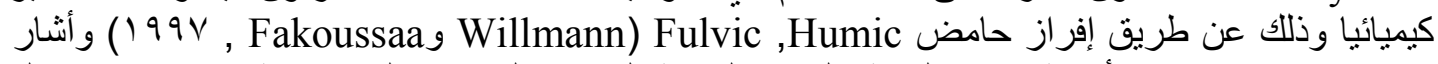
Mishio

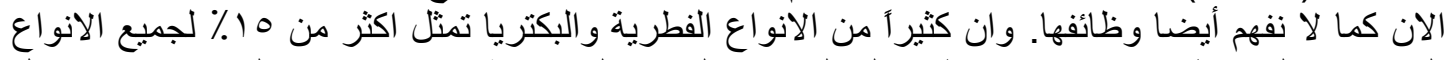

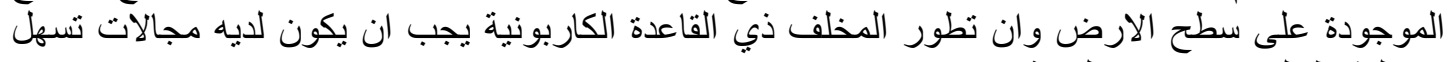
من النشاط المايكروي في التربة التربة

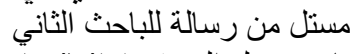

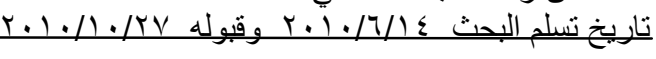




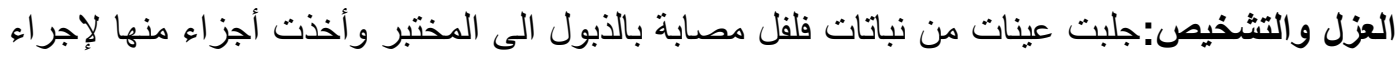

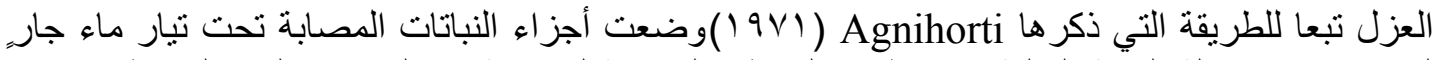

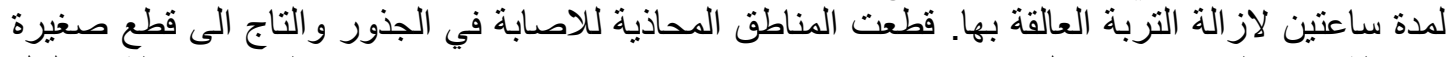

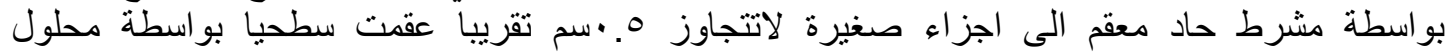

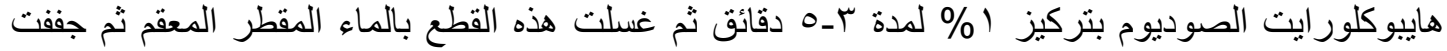

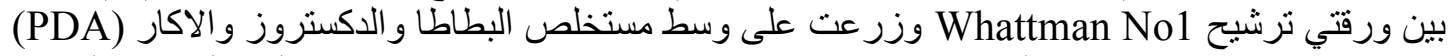
Potato Dexrose Agar

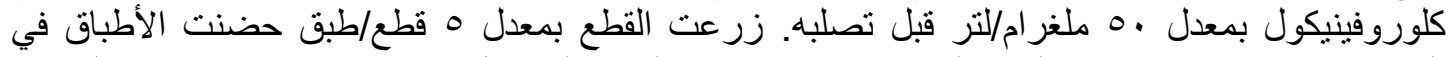

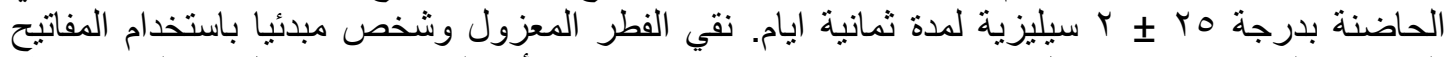

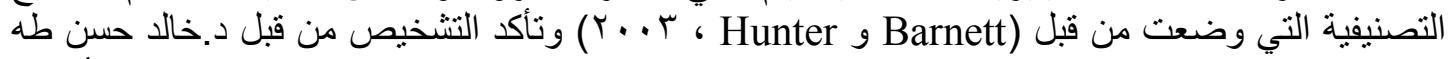

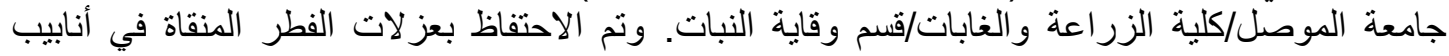

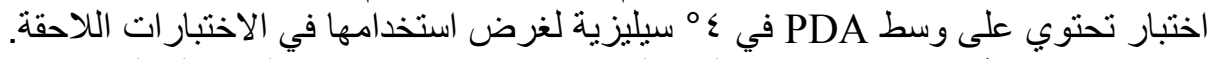
تحضير لقاح الفطر الممرض :حضر لقاح الفطر F.o.c باستخدام بذور الدخن المحلي

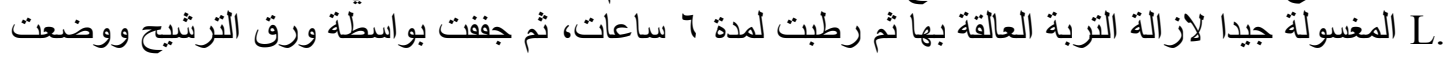

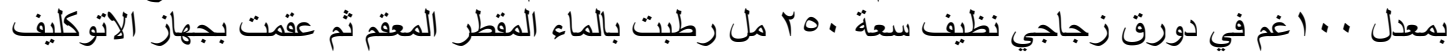

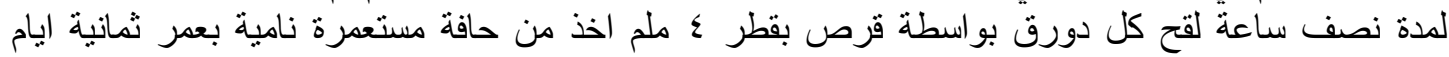

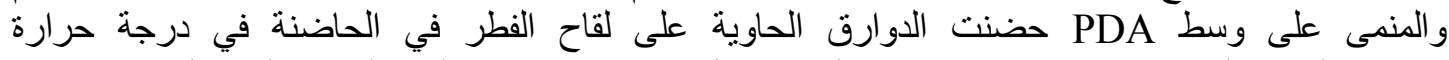

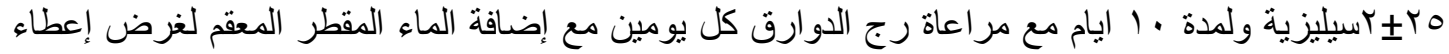

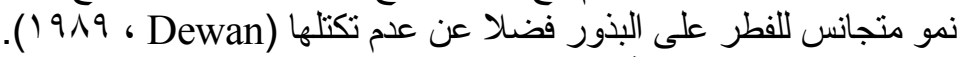

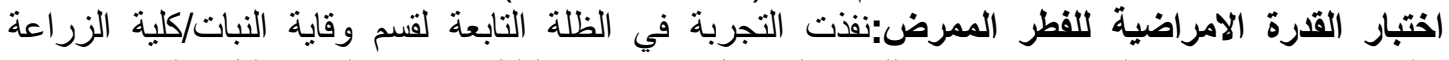

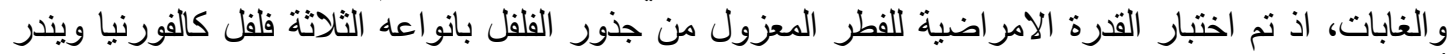

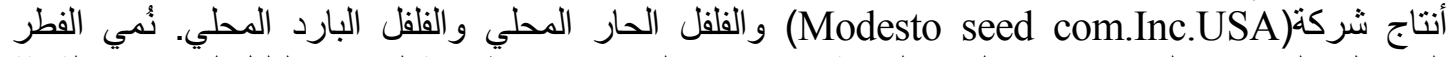

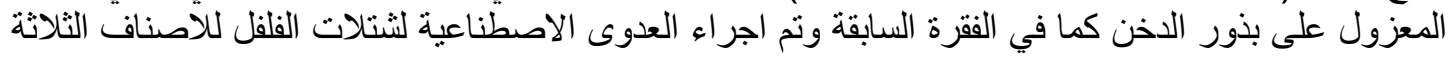

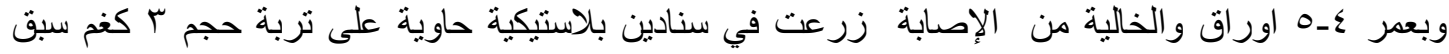

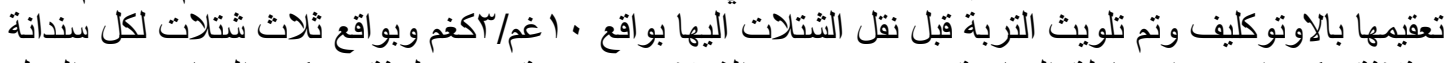

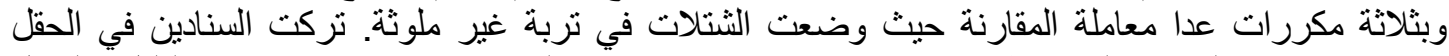

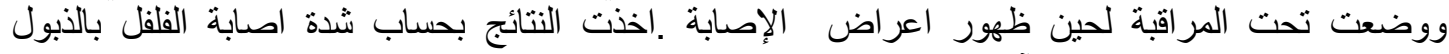
الفيوزارمي تبعا لمقياس Gao وآخرون ( 990 ( ) المؤلف من خمسة درجات وكما ياتي :
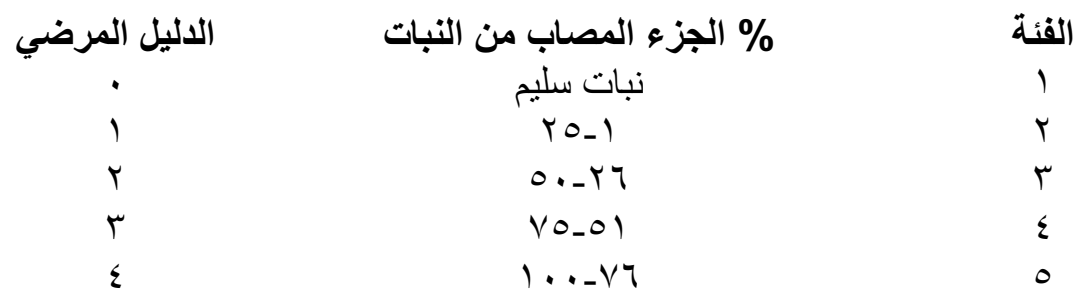

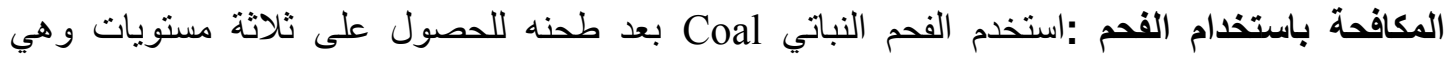

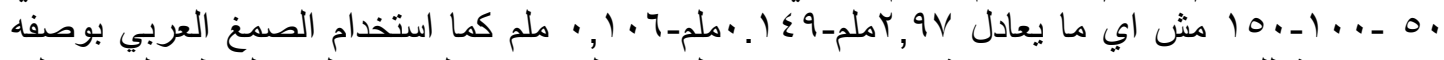

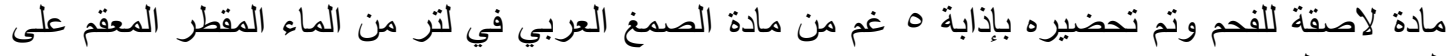
الصنف كاليفورنيا ويندر.

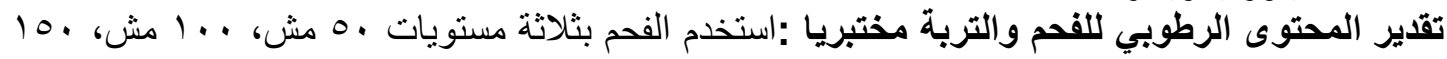

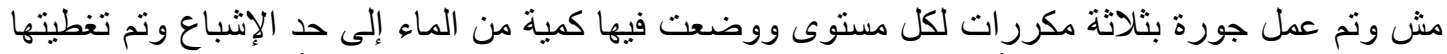

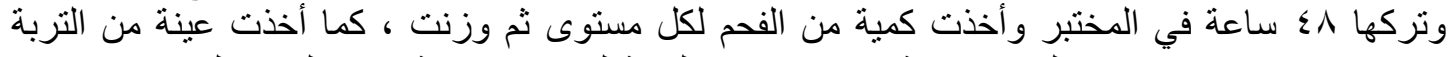

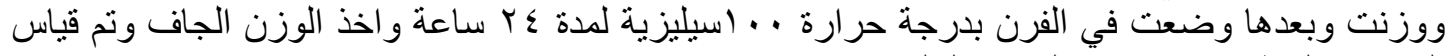
المحتوى الرطوبي باستخدام القانون التالي:

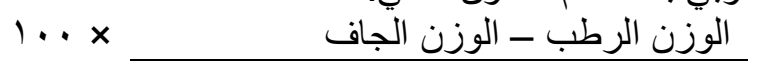


الوزن الجاف - وزن العلبة

مكافحة الذبول الفيوزاريومي في الظلة :نفذت تجربة عاملية وفق تصميم القطاعات العشوائية الكاملة

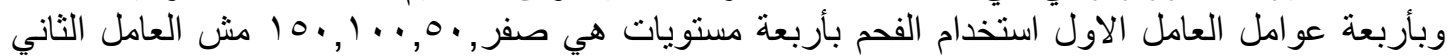

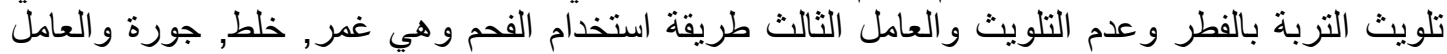

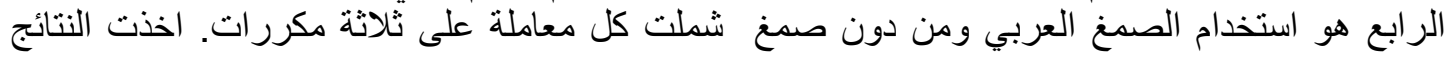

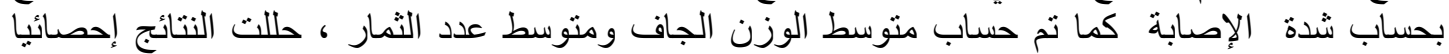
واختبرت متوسطاتها بطريقة دنكن متعدد الحدود.

\section{النتائج والمناقثة}

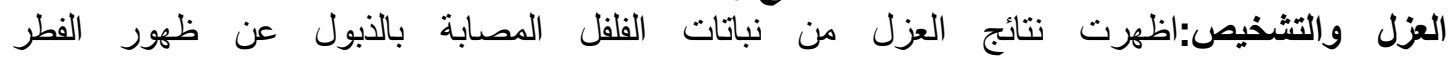
دوكانت مستعمر ات الفطر على وسط PDAsarium oxysporum f.sp. capsici

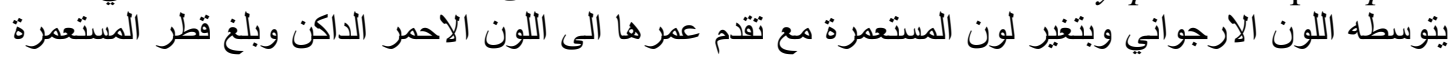

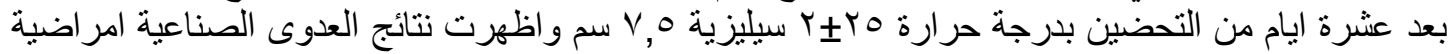

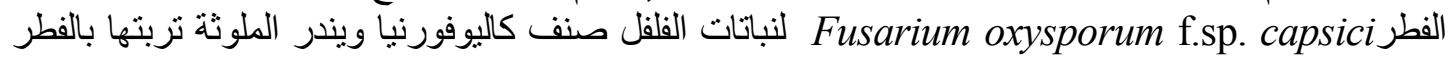
ويعد هذا الفطر هو المسؤول عن إحداث الاصدابات في حقول الفلفل .

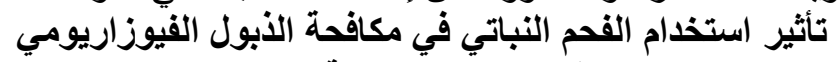

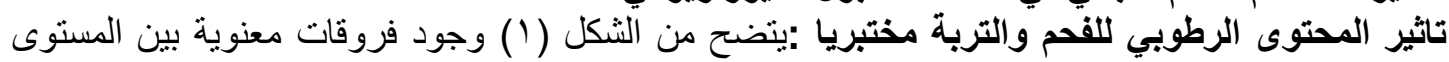

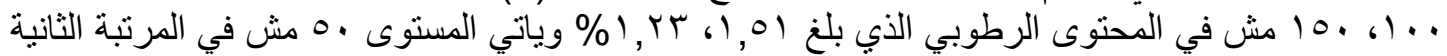

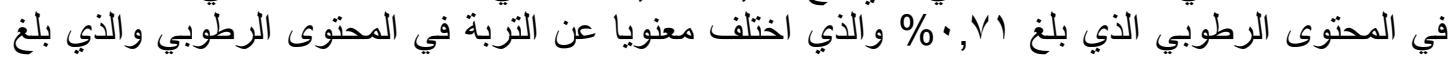

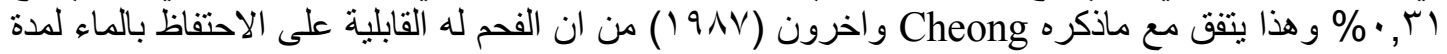

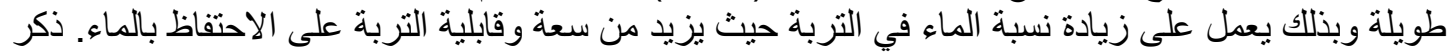
Jorge-Silva

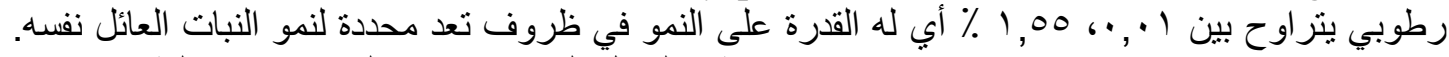

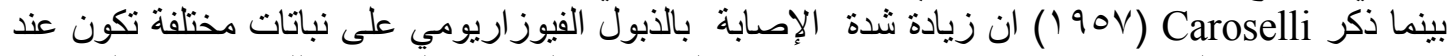

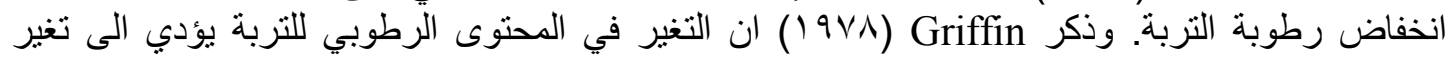
الصفات الفيزيائية و الكيميائية للتربة وبذلك تؤثر وبشكل معنوي في المسببات المرضية في التربة.

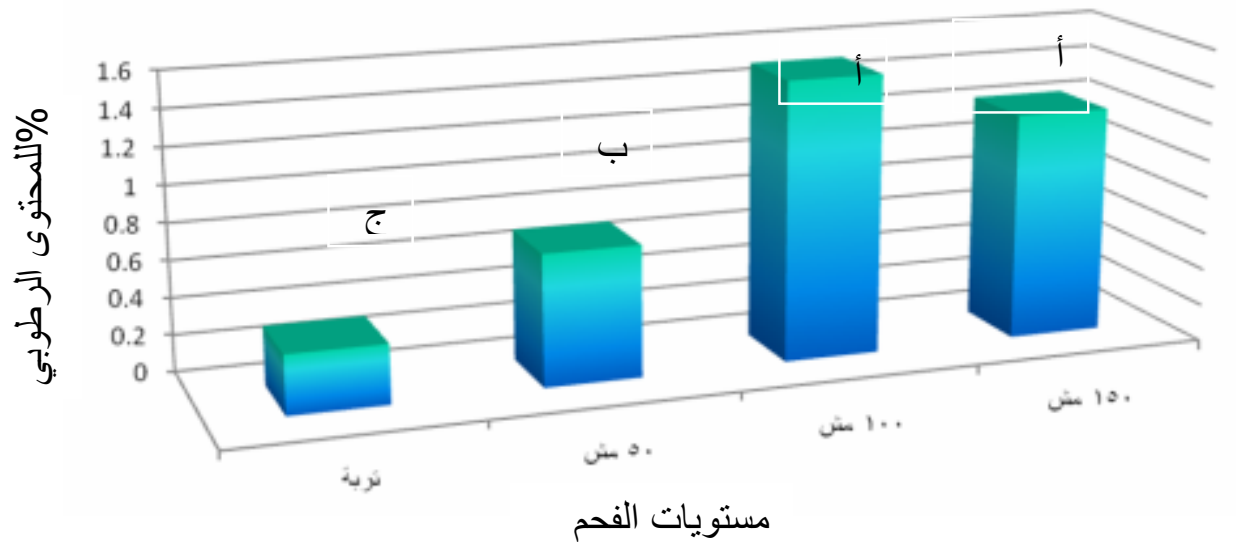

$$
\text { الثكل ( ) تأثثير مستويات الفحم في المحتوى الرطوبي }
$$

تاثير الفحم بمستوياته الثلاثة في مكافحة مرض الأبول في الظلة :استخدمت ثناثة مستويات من الفحم .0.

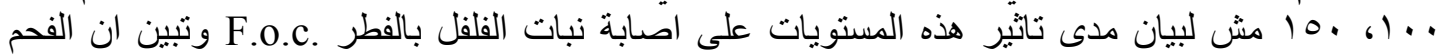

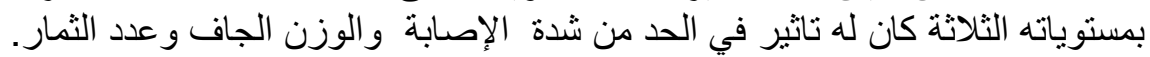

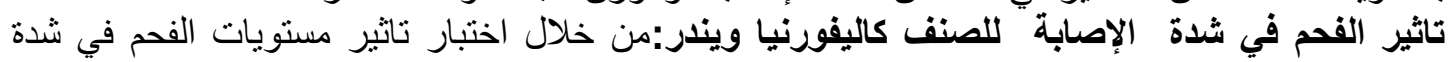

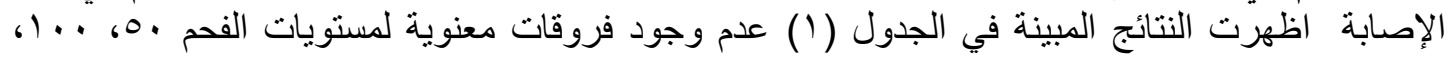




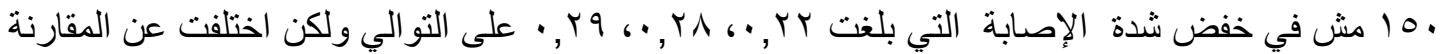

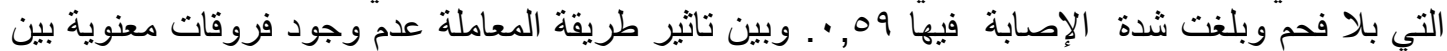

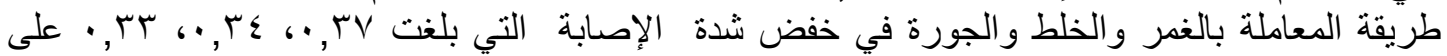

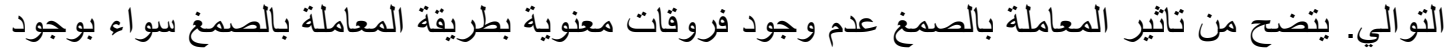

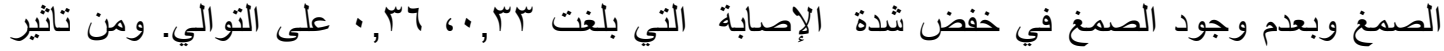

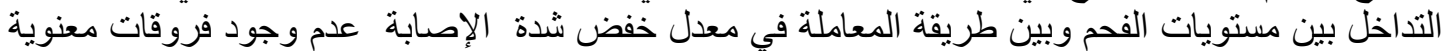

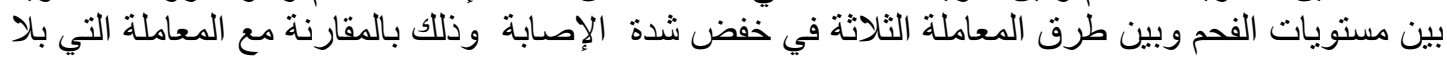

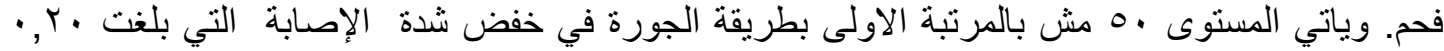

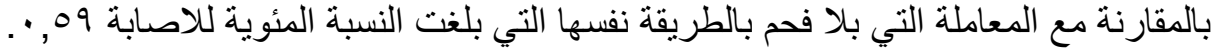

الجدول (1): تأثير الفحم بمستوياته الثلاثة في شدة الإصابة لصنف الفلفل كاليفورنيا ويندر.

\begin{tabular}{|c|c|c|c|c|c|}
\hline \multirow{2}{*}{ تأثنر مستويات } & \multirow{2}{*}{ 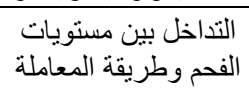 } & \multicolumn{2}{|c|}{ المعاملة بالصمغ } & \multirow{2}{*}{ طريقة المعاملة } & \multirow{2}{*}{ مستويات الفحم } \\
\hline & & بلا صمغ & صمغ & & \\
\hline \multirow{3}{*}{$1 ., 09$} & $1 \cdot, 09$ & $1 ., 09$ & $1 ., 09$ & غمر & \multirow{3}{*}{ صفر } \\
\hline & $1 \cdot, 09$ & $1 ., 09$ & $1 ., 09$ & خلط & \\
\hline & $i \cdot, 09$ & $i ., 09$ & $1 ., 09$ & جورة & \\
\hline \multirow{3}{*}{ } & r r • ب ج & ج• & مار, • ب ج & غمر & \multirow{3}{*}{0.} \\
\hline & ס ז, • ب ج & r & דוץ, ب ج ج & خلط & \\
\hline & ج•,r. & & ج.,10 & جورة & \\
\hline \multirow{3}{*}{ } & & • • • ب ج ج & & غمر & \multirow{3}{*}{$1 \cdots$} \\
\hline & اس, • ب ج & T & وq" أ ب ج & خلط & \\
\hline & ד צ • ب ج & & דוץ, • ب ج & جورة & \\
\hline \multirow{3}{*}{ q } & 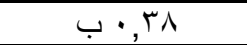 & أ & & غمر & \multirow{3}{*}{10.} \\
\hline & & דוr, • ب ج & ج•,r. & خلط & \\
\hline & ד צ • ب ج & آ, • ب ج & ج. & جورة & \\
\hline & \multirow{4}{*}{ تأثير طريقة المعاملة } & $1 \cdot, 09$ & $1 ., 09$ & صفر & \multirow{4}{*}{ و المعاملة بالصات الفحم بين } \\
\hline & & & & 0. & \\
\hline & & ب & • • • • ب & $1 \cdots$ & \\
\hline & & هر, • ب & & 10. & \\
\hline & $i \cdot, r V$ & $1 \cdot, q$ & I., $\mathrm{I} \varepsilon$ & غمر & \multirow{3}{*}{ 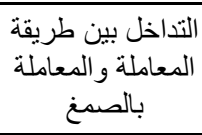 } \\
\hline & 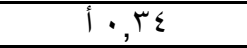 & I. & I. & خلط & \\
\hline & أ & i. & i. & جورة & \\
\hline & & דו, • ו & T. & \multicolumn{2}{|c|}{ المعاملة بالصمغ } \\
\hline
\end{tabular}

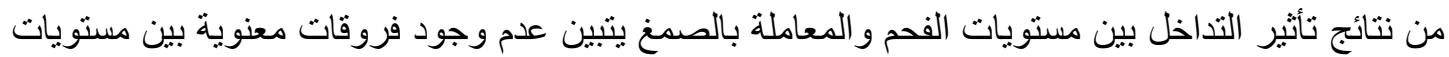

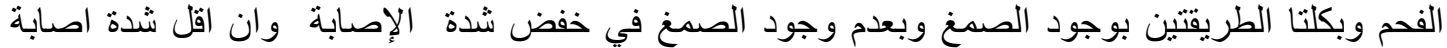

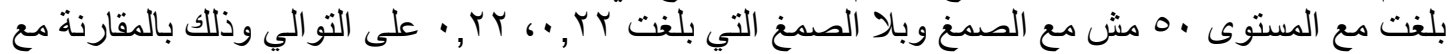

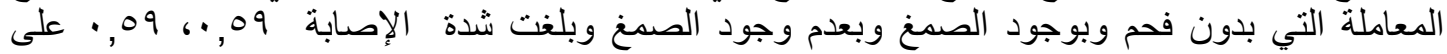

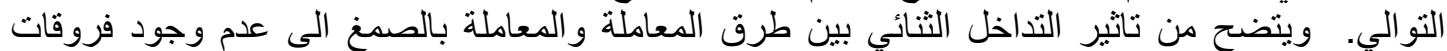

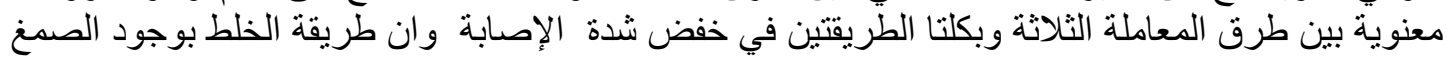

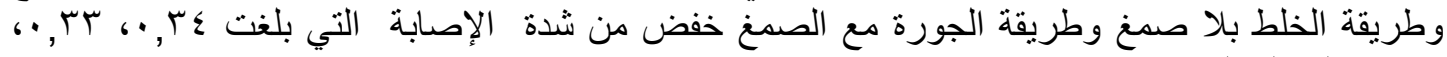

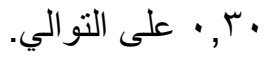
وتبين من نتائج التحليل الإحصائي لتأثير التداخل الثناثي بين مستويات الفحم وبين طريقة المعاملة وبين

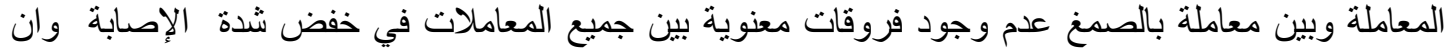

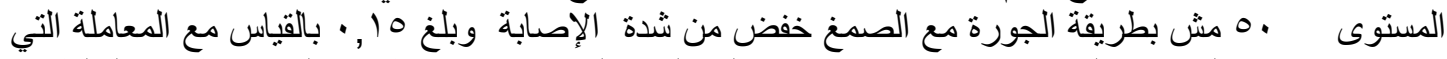

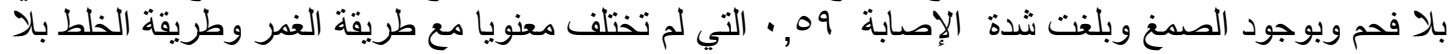

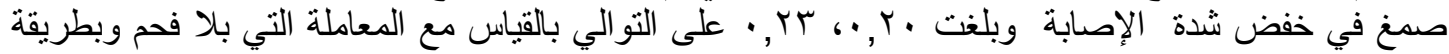

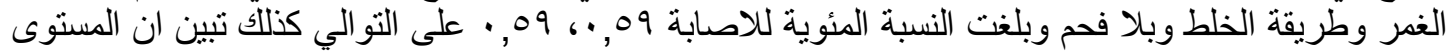




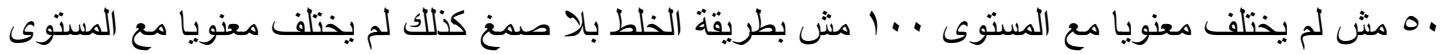

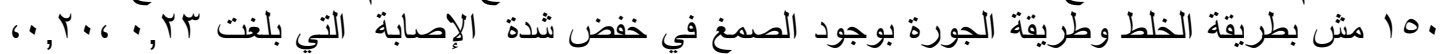

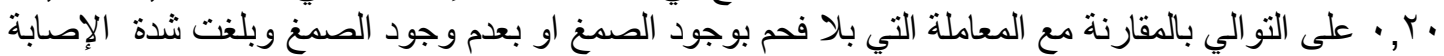

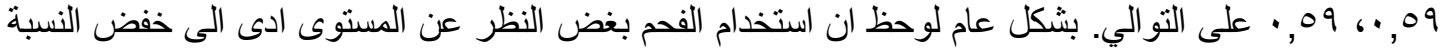

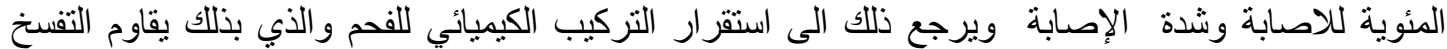

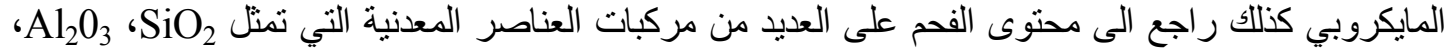
، $\mathrm{CaO}$

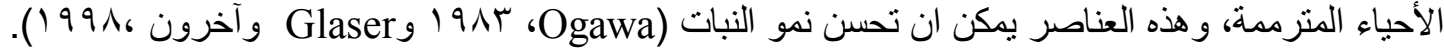

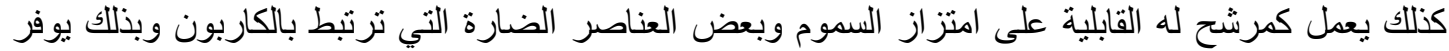

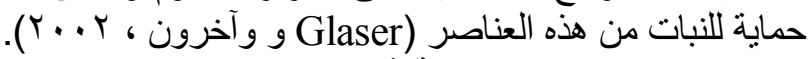

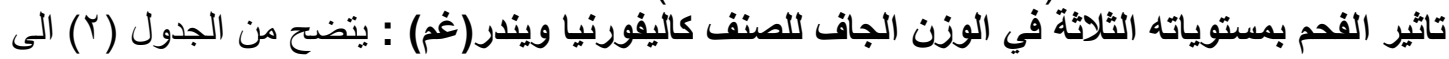

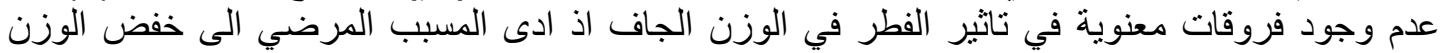

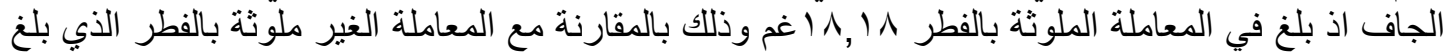

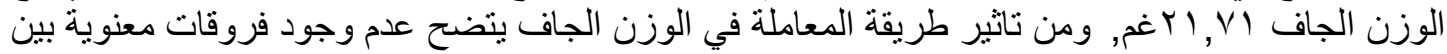

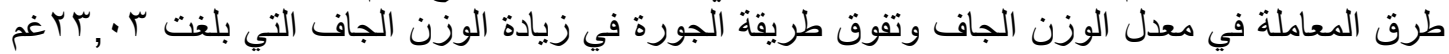

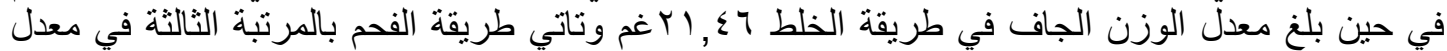

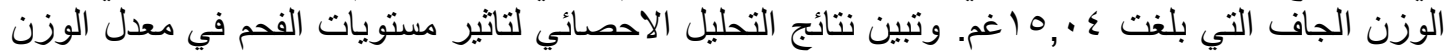

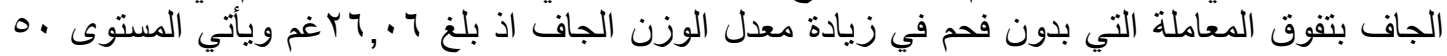

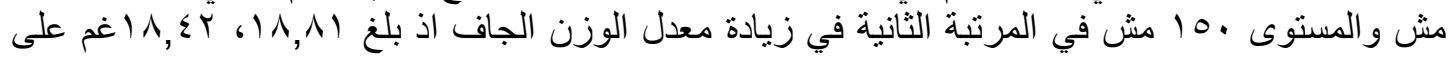

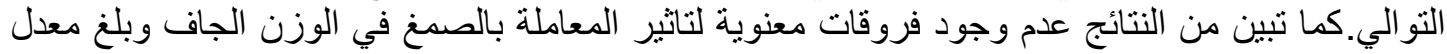

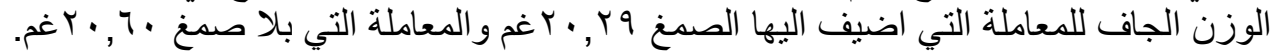

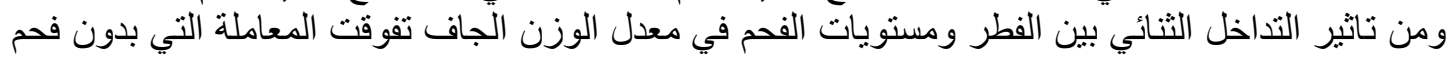

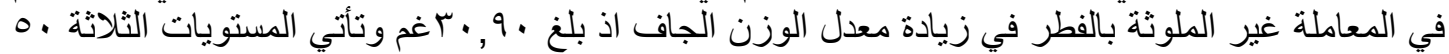

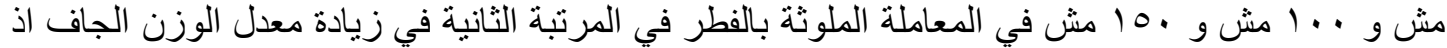

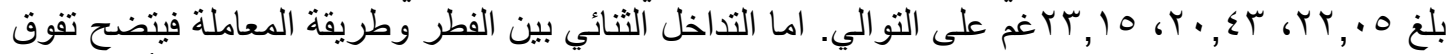

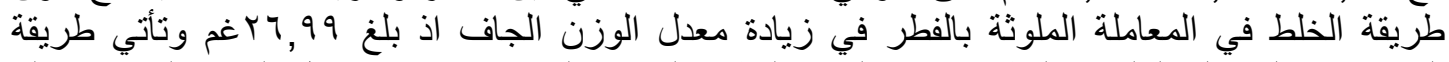

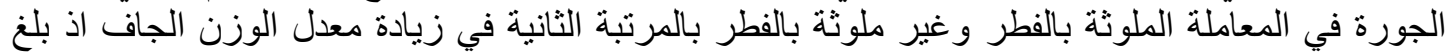

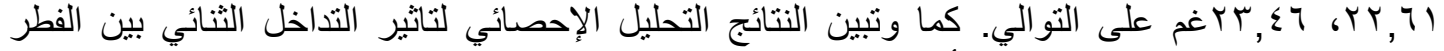

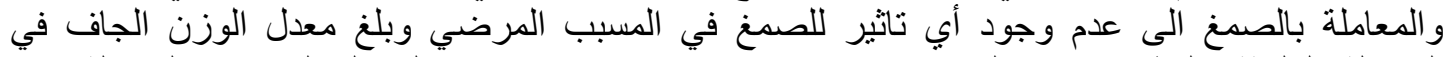

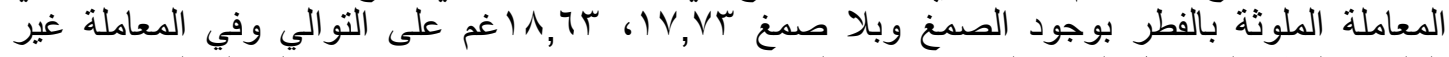

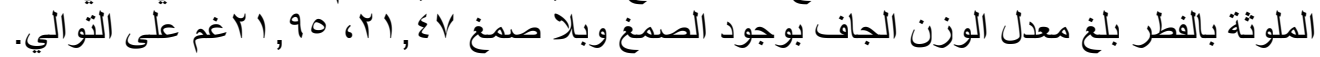

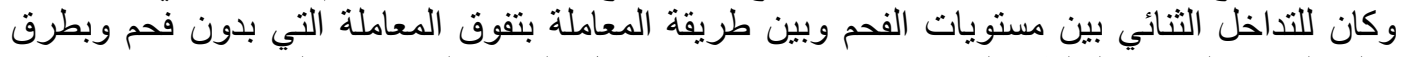

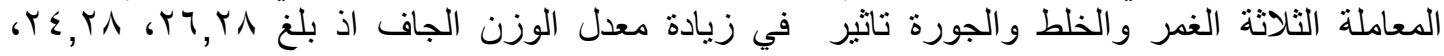

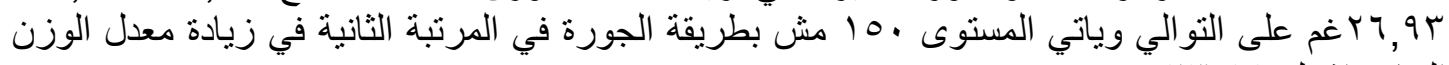

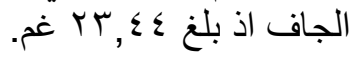




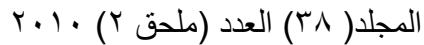
(ISSN 1815-316x)

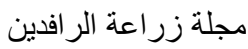

الجدول (Y) تأثير الفحم بمستوياته الثناثة في عدد الثمار لصنف الفلفل كاليفورنيا ويندر

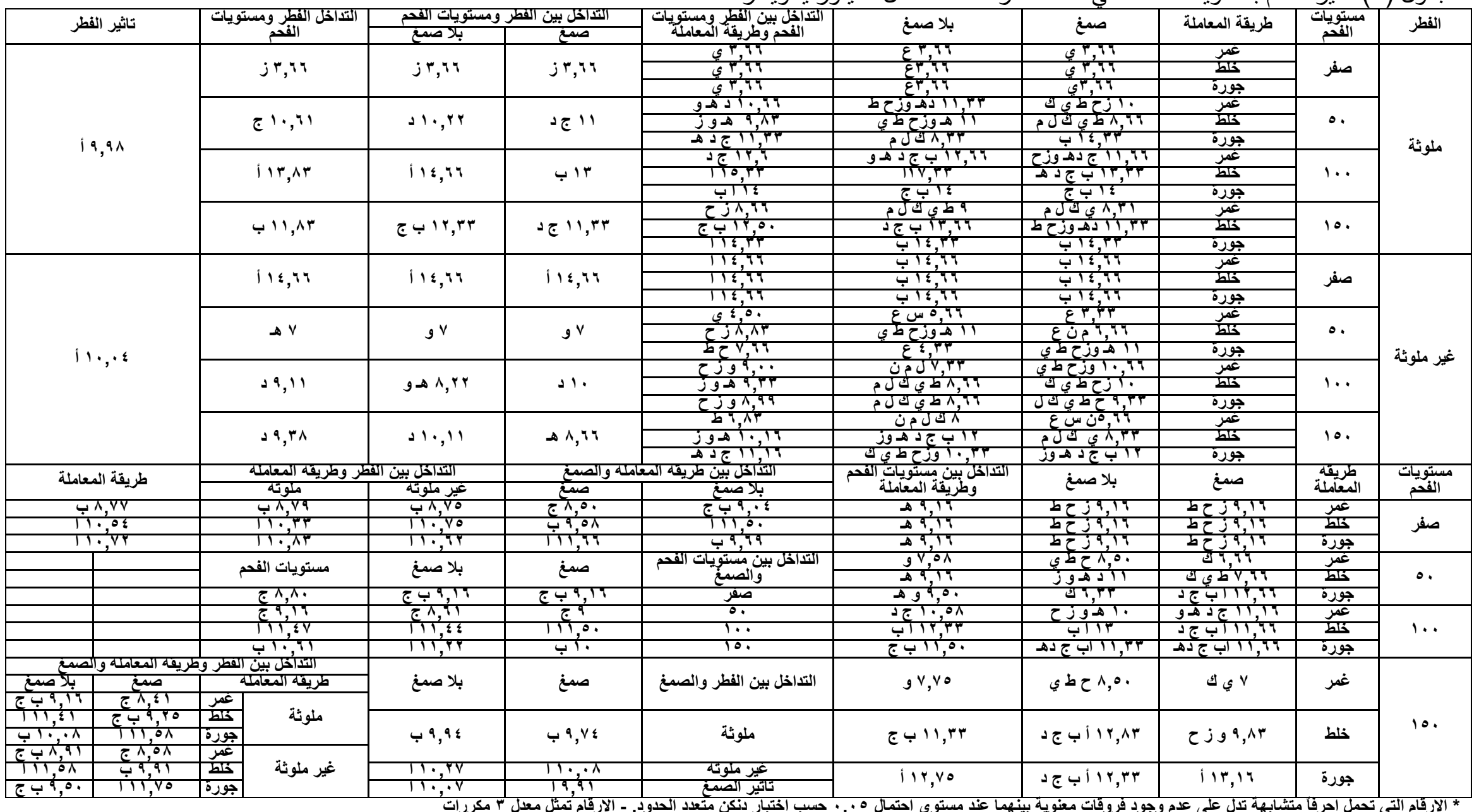




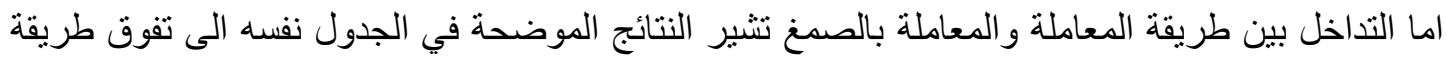

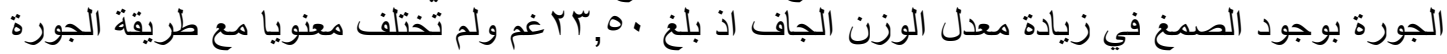

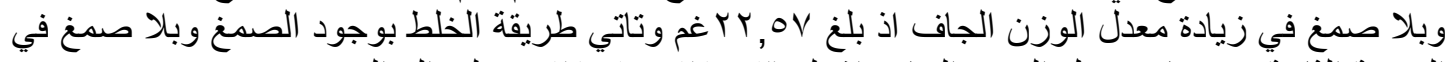

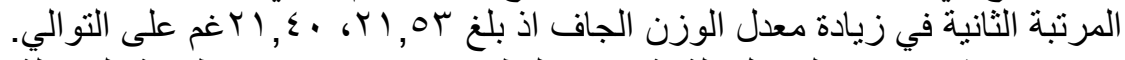

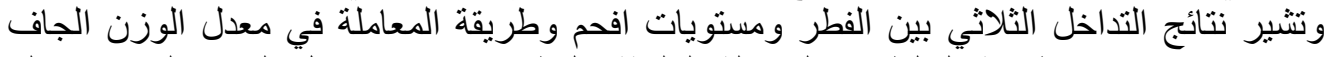

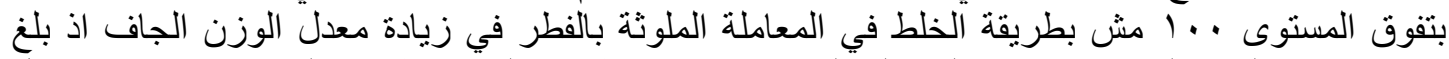

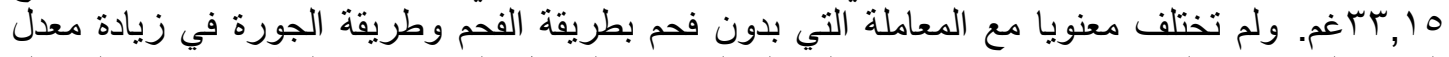

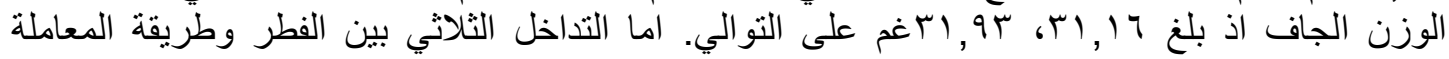

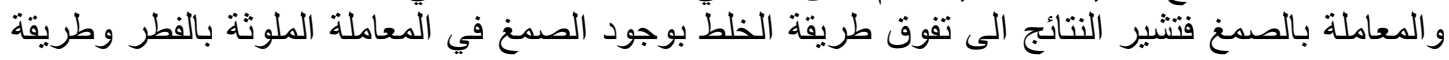

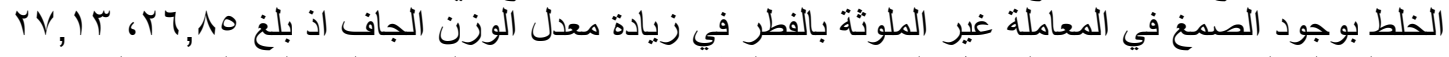

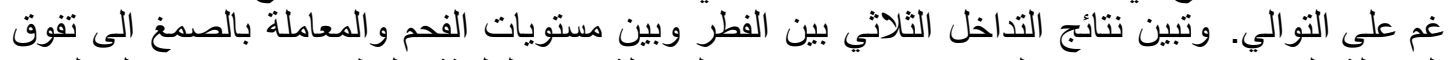

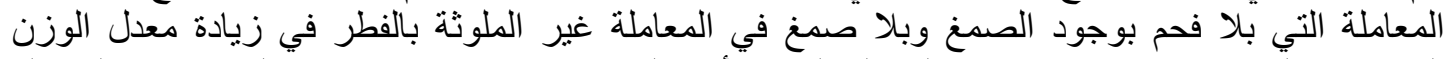

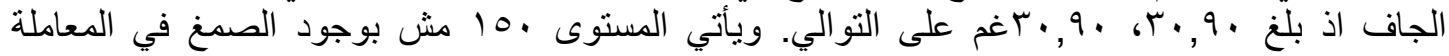

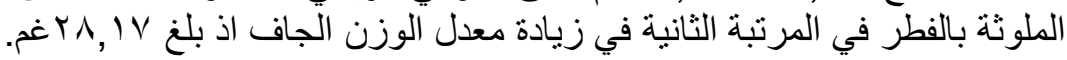

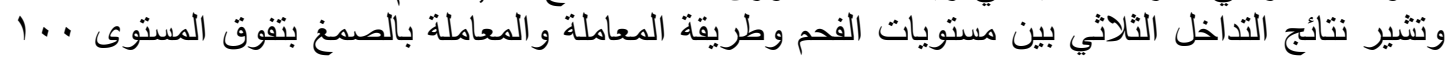

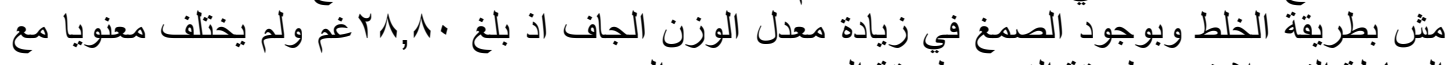

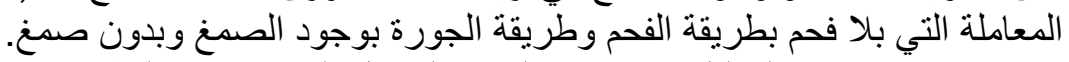

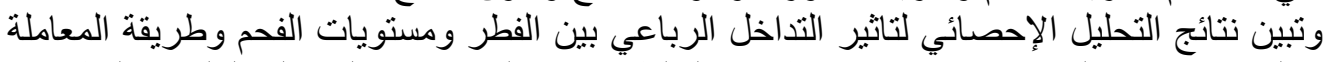

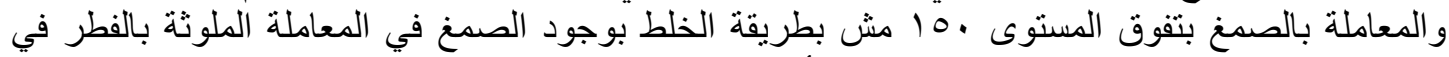

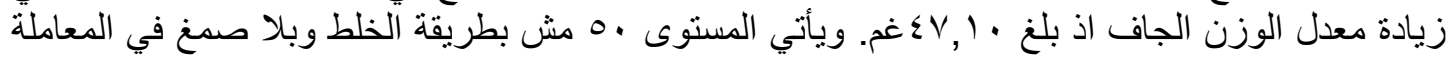

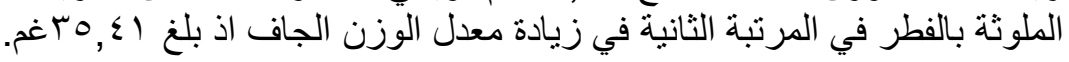

\section{USING COAL TO REDUSING FUSARMIC WILT CAUSED BY FUSARIUM OXYSPORUM F.SP. CAPSICI ON PEPPER}

Nidhal Y. Mohammed Al-Morad Alaa H. Al-Khafagi

Dept. of Plant Prot. College of Agric and Forestry. University of Mosul .Iraq

\section{ABSTRACT}

The study was conducted at the Department of plant protection, College of Agriculture and forestry, University of Mosul, during the growing season of 2007 2008. The result of field survey which was carried out on pepper field in A1Rashedia and al Gayara / Ninevah governorate showed that pepper wilt disease was caused by Fusarium oxysporum f.sp. capsici .Pathogensity test showed that the fungi caused total wilt to the pepper plants for verity California Wonder. In greenhouse experiment , adding coal to the planting soil with three grade $(50,100,150 \mathrm{mish})$ ) caused significant decrease in diseases severity which were $0.22,0.28,0.29$ respectively comparing with control treatment 0.59.In greenhouse experiment, adding coal to the soil with three fining grade $(50,100,150 \mathrm{mish})$ and three different type of application mixing with planting soil , direct application in planting site and soil drenching with or without gum had improved all growth parameter that were studied compeering with control treatment.

\section{المصادر}

Agnihorti , V.P. (1971). Effects of Certain fungi toxicants on the viability and pathogenicity of sclerotia of waitea circinata Phytopath., 70:71-80.

Agrios, G.N. (1997). Blant Pathology. Academic Press. New York. 568pp.

Anderson, W.C. (1985). Tissue culture propagtion of red and black vaspberries Rubus idaeus and R. occidentalis. Hort. Sci. 23:132. 


$$
\text { مجلة زر اعة الر افدين }
$$

Barnett, H. Land, B.B. Hunter (2003) Illustated genera of imperfect fungi. The American Phytopathological Society, 3340 Pilot Knob Road St. Paul , Minnesota 55121-2097, USA. 217pp.

Beckman, C.H. (1987). The Nature of Wilt Diseases. St. Paul MN:APS press.

Caroselli, N.E. (1957) Verticillium wilt of maples. R.I. Univ. Agric. Exp. Stn. Bull. 3335: $1-84$.

Cheong, S.T.; S.K. Kim; K.Y. Pack and H.K. Ahn (1987). In Vitro rooting and branching responses of Jujube shoots as affected by growth regulators. J. Kor. Soc. Hort. Sci. 28 : 53-60.

Dewan , M.M. (1989). Identity and frequency of occurence of fungi in roots of wheat and rye grass and their effect on take all and host growth. Ph. D. Thesis Univ. Wes. Australia pp. 210.

Gao, H.; G.H., Beckman and W.C., Mueller. (1995). The rate of vascular colonization as a measure of the gencty pic niteration between various cultivars of tomato and various formae or races of Fusarium oxysporum, Physiol. Mol. plant pathol. 46;29-43.

Garrett , S.D. (1970). Root Disease and Soil Borne Pathogen. Cambridge Univ. Press. $252 \mathrm{pp}$.

Glaser, B.; L. Haumaier; G. Guggenberger and W. Zeekh (1998). Black Carbon in soil. The use of benzene carboxylic acids as specific markers. org Geochem. 29:811:819.

Glaser, B.; J. Lehmann and W. Zeeh (2002). Ameliorating physical and chemical properties of highly weathered soils in the tropics with charcoal-areview. Biol. and Fert. Soil, 35:219-230.

Gonsalvas, A.K. and S.R. Ferreira (1994). "Fusarium Primmer" University of Hawaii Press, Manoa. Data sheet.

Griffin, D.M. (1978) Effect of soil moisture on survival and spread of pathogens. Pages 175-197 in: Water Deficits and Palnt Growth T.T. Kozlowski ed. New York: Academic press USA.

Jorge-Silva, M.A.; M.L. Rodrigues; J.F.P. Ferraz and C.P.P. Ricardo (1989). Effect of water availability on growth of fusarium oxysporum $f$. sp. Melonia and no host-parasite interactions. Myco. Res., 2: 157-161.

Laborda, F.; M. Fernandez; N. Luna and I.F. Monistral (1997). Study of the mechanisms by which microorganisms solubilize and/or liquefy spanish coals fue/proc Technol. 52:95-107.

Mishio, M. (1999). Microbial fertilizers in Japan, Bulletin by National Institute of Agro-environmental Sciences, Japan.

Ogawa, M. (1983). Effect of Charcoal on the root nodule and VA mycorrhizal formation of soybean. Proceedings of the Third-inter. Myco. Con. Tok. Jap. 578pp.

Sanago, S. and G. Carpenter (2007). Geographical Distribution and Causal Agents of Chile Pepper Wilt in New Mexico. State University, Plant Pathol. 789pp.

Starrantina, A. and I. Caruso (1987). Experiences on the in vitro propagation of some citrus rootstocks. Acta. Hort. 212:471-478.

Willmann, G. and R.M. Fakoussaa (1997). Biological bleaching of watwe solube macromolecules by a basidiomycete strain. Appl. Micr. Biot. 47:95-101. 